Kragujevac Journal of Mathematics

Volume 39(2) (2015), Pages 173-182.

\title{
GALILEAN GEOMETRY OF CORRESPONDING SURFACES TO PRODUCTION MODELS IN ECONOMICS
}

\author{
M. E. AYDIN ${ }^{1}$ AND S. A. SEPET ${ }^{2}$
}

\begin{abstract}
In this paper we study the curvature properties of the corresponding surfaces in the Galilean 3-space $\mathbb{G}_{3}$ to some production models in economics such as the generalized Cobb-Douglas, the generalized ACMS and the Allen production functions. We classify such surfaces of null curvature in $\mathbb{G}_{3}$.
\end{abstract}

\section{INTRODUCTION}

In economics, a production function is a mathematical expression which denotes the physical relations between the output generated by a firm, an industry or an economy and the inputs that have been used. Explicitly, a production function is a map that has non-vanishing first derivatives defined by

$$
f: \mathbb{R}_{+}^{n} \longrightarrow \mathbb{R}_{+},\left(x_{1}, x_{2}, \ldots, x_{n}\right) \longmapsto f\left(x_{1}, x_{2}, \ldots, x_{n}\right),
$$

where $f$ is the quantity of output, $n$ is the number of inputs and $x_{1}, x_{2}, \ldots, x_{n}$ are the inputs (such as capital, labor, raw materials etc.).

Almost all economic theories presuppose a production function, either on the firm level or the aggregate level. In this sense, the production function is one of the key concepts of mainstream neoclassical theories. By assuming that the maximum output technologically possible from a given set of inputs is achieved, economists using a production function in analysis are abstracting from the engineering and managerial problems inherently associated with a particular production process.

In order for the production functions to model economic reality, they are required to have some properties (see e.g. $[8,31]$ ). One of the most important of these properties

\footnotetext{
Key words and phrases. Generalized Cobb-Douglas production function, generalized ACMS production function, Allen production function, Galilean space, Gaussian curvature, mean curvature.

2010 Mathematics Subject Classification. Primary: 91B38. Secondary: 53A35, 53B25.

Received: September 1, 2015.

Accepted: October 29, 2015.
} 
is that the production function $f$ is to be homogeneous, i.e. there exists a real number $p$ such that

$$
f\left(\lambda x_{1}, \lambda x_{2}, \ldots, \lambda x_{n}\right)=\lambda^{p} f\left(x_{1}, x_{2}, \ldots, x_{n}\right), \quad \lambda \in \mathbb{R}_{+},
$$

which means that when the inputs are multiplied by the same factor, the output is multiplied by some power of the factor.

If $p<1$ (resp. $p>1$ ) in (1.2), then the production function is said to have decreasing (resp. increasing) return to scale. If $p=1$, then it is said to have constant return to scale.

The presence of increasing returns means that a one percent increase in the usage levels of all inputs would result in a greater than one percent increase in output; the presence of decreasing returns means that it would result in a less than one percent increase in output. Constant returns to scale is the in-between case (cf. [11]).

For the production function given by (1.1), the elasticity of production with respect to a certain factor $x_{i}$ is defined as

$$
E_{x_{i}}=\frac{x_{i}}{f} f_{x_{i}}, \quad f_{x_{i}}=\frac{\partial f}{\partial x_{i}}, \quad i \in\{1, \ldots, n\} .
$$

A. D. Vilcu and G. E. Vilcu [35] completely classified the homogeneous production functions with constant proportional marginal rate of substitution.

The most famous production function is Cobb-Douglas (CD) production function, introduced in 1928 by C. W. Cobb and P. H. Douglas [16]. In original form, it is given as

$$
Y=b L^{k} C^{1-k}
$$

where $b$ presents the total factor productivity, $Y$ the total production, $L$ the labor input and $C$ the capital input.

The generalized $C D$ production function of $n$ variables is defined by

$$
f\left(x_{1}, x_{2}, \ldots, x_{n}\right)=A x_{1}^{\alpha_{1}} x_{2}^{\alpha_{2}} \cdots x_{n}^{\alpha_{n}},
$$

where $A, \alpha_{1}, \alpha_{2}, \ldots, \alpha_{n}>0$.

In 1961, K. J. Arrow, et al. [2] introduced a two-factor CES (constant elasticity of substitution) production function given by

$$
Y=A \cdot\left(a K^{r}+(1-a) L^{r}\right)^{\frac{1}{r}},
$$

where $Y$ is the output, $A$ the factor productivity, $a$ the share parameter, $K$ and $L$ the primary production factors, $r=(s-1) / s$ and $s=1 /(1-r)$ is the elasticity of substitution.

The generalized ACMS (or CES) production function of $n$ variables is given by

$$
f\left(x_{1}, x_{2}, \ldots, x_{n}\right)=A\left(a_{1}^{\rho} x_{1}^{\rho}+a_{2}^{\rho} x_{2}^{\rho}+\cdots+a_{n}^{\rho} x_{n}^{\rho}\right)^{\frac{\gamma}{p}},
$$

where $\rho \neq 0, \rho<1, \gamma, A>0$, and $\alpha_{i}>0$ for all $i \in\{1, \ldots, n\}$.

Note that the generalized CD and the generalized ACMS production functions are homogeneous of degree $\sum_{i=1}^{n} \alpha_{i}$ and $\gamma$, respectively. 
The most common quantitative indices of production factor substitutability are forms of the elasticity of substitution. R.G.D. Allen and J.R. Hicks [1] suggested two generalizations of Hicks' original two variable elasticity concept.

The first concept, called Hicks elasticity of substitution, is defined as follows.

Let $f\left(x_{1}, \ldots, x_{n}\right)$ be a production function. Then Hicks elasticity of substitution of the $i$-th production variable with respect to the $j$-th production variable is given by

$$
H_{i j}(\mathbf{x})=-\frac{\frac{1}{x_{i} f_{x_{i}}}+\frac{1}{x_{j} f_{x_{j}}}}{\frac{f_{x_{i} x_{i}}}{\left(f_{x_{i}}\right)^{2}}-\frac{2 f_{x_{i} x_{j}}}{f_{x_{i}} f_{x_{j}}}+\frac{f_{x_{j} x_{j}}}{\left(f_{x_{j}}\right)^{2}}},
$$

where $\mathbf{x}=\left(x_{1}, \ldots, x_{n}\right) \in \mathbb{R}_{+}^{n}, i, j=1, \ldots, n$, and $i \neq j$.

L. Losonczi [23] classified homogeneous production functions of 2 variables, having constant Hicks elasticity of substitution. Then, the classification of L. Losonczi was extended to $n$ variables by B-Y. Chen [13].

The second concept, investigated by R.G.D. Allen and H. Uzawa [32], is the following:

Let $f\left(x_{1}, \ldots, x_{n}\right)$ be a production function. Then the Allen elasticity of substitution of the $i$-th production variable with respect to the $j$-th production variable is defined by

$$
A_{i j}(\mathbf{x})=-\frac{x_{1} f_{x_{1}}+x_{2} f_{x_{2}}+\cdots+x_{n} f_{x_{n}}}{x_{i} x_{j}} \frac{D_{i j}}{D}, \quad \mathbf{x} \in \mathbb{R}_{+}^{n}, \quad i, j=1, \ldots, n, \quad i \neq j,
$$

where $D$ is the determinant of the bordered Hessian matrix

$$
H(f)=\left(\begin{array}{cccc}
0 & f_{x_{1}} & \cdots & f_{x_{n}} \\
f_{x_{1}} & f_{x_{1} x_{1}} & \cdots & f_{x_{1} x_{n}} \\
\vdots & \vdots & \cdots & \vdots \\
f_{x_{n}} & f_{x_{1} x_{n}} & \cdots & f_{x_{n} x_{n}}
\end{array}\right)
$$

and $D_{i j}$ is the co-factor of the element $f_{x_{i} x_{j}}$ in the determinant $D(D \neq 0$ is assumed). The authors in [6] called such matrices the Allen's matrix and classified some production functions by using the singularity of the Allen matrices [3-6].

It is a simple calculation to show that in the case of two variables Hicks elasticity of substitution coincides with Allen elasticity of substitution.

In economics, goods that are completely substitutable with each other are called perfect substitutes. Mathematically, a production function is a perfect substitute if it is of the form:

$$
f\left(x_{1}, \ldots, x_{n}\right)=\sum_{i=1}^{n} a_{i} x_{i},
$$

where $a_{1}, \ldots, a_{n}$ are nonzero constants [15]. 
On the other hand, G. E. Vilcu [33] established an interesting link between some fundamental notions in the theory of production functions and the differential geometry of corresponding hypersurfaces to the graphs of such functions in the Euclidean $(n+1)$-spaces $\mathbb{E}^{n+1}$. The author proved that the generalized CD production function has constant return to scale if and only if the corresponding hypersurface is developable. The author joint with A. D. Vilcu [34] generalized this result to the case of the generalized CES production function.

Later, X. Wang and Y. Fu [36] obtained a non-existence result for flat graph hypersurfaces of the generalized CD production functions and also proved that a graph hypersurface of the generalized ACMS production function is minimal in $\mathbb{E}^{n+1}$ if and only if it is a perfect substitute.

Most recently, B.-Y. Chen, et al. [10,17] investigated the graph hypersurfaces of the production models via the isotropic geometry. For further study of graph hypersurfaces of production functions we refer the reader to [3-6, 9-15].

The present paper deals with the curvature properties of the corresponding surfaces in the Galilean 3-space $\mathbb{G}_{3}$ to some production models in economics such as the generalized CD, the generalized ACMS and the Allen production functions. We classify such surfaces with null Gaussian and mean curvature in $\mathbb{G}_{3}$.

\section{Basics on Galilean GeOMetry}

For later use, we provide a brief review of Galilean geometry from [7, 18, 19, 21, 25, $27-30,37]$.

The Galilean geometry is one model of the real Cayley-Klein geometries which has projective signature $(0,0,+,+)$. The absolute figure of the Galilean geometry is an ordered triple $\{\omega, f, I\}$, where $\omega$ is the ideal (absolute) plane, $f$ a line in $\omega$ and $I$ is the fixed eliptic involution of the points of $f$. The homogeneous coordinates in $\mathbb{G}_{3}$ is introduced in such a way that the ideal plane $\omega$ is given by $x_{0}=0$, the ideal line $f$ by $x_{0}=x_{1}=0$ and the elliptic involution by

$$
\left(0: 0: x_{2}: x_{3}\right) \longrightarrow\left(0: 0: x_{3}:-x_{2}\right) \text {. }
$$

By means of the affine coordinates defined by $\left(x_{0}: x_{1}: x_{2}: x_{3}\right)=(1: x: y: z)$, the similarity group $H_{8}$ of the Galilean space $\mathbb{G}_{3}$ has the following form

$$
\begin{aligned}
& \bar{x}=a+b x \\
& \bar{y}=c+d x+r(\cos \theta) y+r(\sin \theta) z \\
& \bar{z}=e+f x+r(-\sin \theta) y+r(\cos \theta) z,
\end{aligned}
$$

where $a, b, c, d, e, f, r$, and $\theta$ are real numbers. In particular, for $b=r=1$, the group becomes the group of isometries (proper motions), $B_{6} \subset H_{8}$, of the Galilean space $\mathbb{G}_{3}$.

A plane is called Euclidean if it contains $f$, otherwise it is called isotropic, i.e. the planes $x=c$, where $c$ is a constant, are Euclidean, in particular the plane $\omega$. Other planes are isotropic. 
We introduce the metric relations with respect to the absolute figure. The Galilean distance between the points $P_{i}=\left(u_{i}, v_{i}, w_{i}\right),(i=1,2)$, is given by

$$
d\left(P_{1}, P_{2}\right)= \begin{cases}\left|u_{2}-u_{1}\right|, & \text { if } u_{1} \neq 0 \text { or } u_{2} \neq 0 \\ \sqrt{\left(v_{2}-v_{1}\right)^{2}+\left(w_{2}-w_{1}\right)^{2}}, & \text { if } u_{1}=0 \text { and } u_{2}=0\end{cases}
$$

The Galilean scalar product between two vectors $\mathbf{X}=\left(x_{1}, x_{2}, x_{3}\right)$ and $\mathbf{Y}=\left(y_{1}, y_{2}, y_{3}\right)$ is given by

$$
\mathbf{X} \cdot \mathbf{Y}= \begin{cases}x_{1} y_{1}, & \text { if } x_{1} \neq 0 \text { or } y_{1} \neq 0 \\ x_{2} y_{2}+x_{3} y_{3}, & \text { if } x_{1}=0 \text { and } y_{1}=0\end{cases}
$$

In this sense, the Galilean norm of a vector $\mathbf{X}$ is $\|\mathbf{X}\|=\sqrt{\mathbf{X} \cdot \mathbf{X}}$. A vector $\mathbf{X}=$ $\left(x_{1}, x_{2}, x_{3}\right)$ is called isotropic if $x_{1}=0$, otherwise it is called non-isotropic.

The cross product in the sense of Galilean space is

$$
\mathbf{X} \times_{\mathbb{G}} \mathbf{Y}=\left(0,-\left|\begin{array}{ll}
x_{1} & x_{3} \\
y_{1} & y_{3}
\end{array}\right|,\left|\begin{array}{ll}
x_{1} & x_{2} \\
y_{1} & y_{2}
\end{array}\right|\right)
$$

Let $D$ be an open subset of $\mathbb{R}^{2}$ and $M^{2}$ a surface in $\mathbb{G}_{3}$ parameterized by

$$
\mathbf{r}: D \longrightarrow \mathbb{G}_{3}, \quad(u, v) \longmapsto \mathbf{r}(x, y)=(x, y, z(x, y)),
$$

where $z$ is a smooth and real-valued function on $D$. Such surfaces are well-known Monge surfaces and always admissible (i.e. without Euclidean tangent planes).

The Gaussian curvature $K$ and the mean curvature $H$ of $M^{2}$ in $\mathbb{G}_{3}$ are given by

$$
K=\frac{z_{x x} z_{y y}-z_{x y}^{2}}{\left(1+\left(z_{y}\right)^{2}\right)^{2}} \quad \text { and } \quad H=\frac{z_{y y}}{2\left(1+\left(z_{y}\right)^{2}\right)^{\frac{3}{2}}} .
$$

A surface in $\mathbb{G}_{3}$ is said to be minimal (resp. flat) if its mean curvature (resp. Gaussian curvature) vanishes.

Notice that a surface of the form (2.1) corresponds to the graph of the function $z(x, y)$ and it is flat if and only if the function $z(x, y)$ satisfies the homogeneous Monge-Ampère equation, i.e. $z_{x x} z_{y y}-z_{x y}^{2}=0$.

\section{Corresponding Surfaces to Production Functions}

In this section, we focus on the curvature properties of the corresponding graph surfaces to some production functions such as the CD, the ACMS, the Allen production functions.

We consider such surfaces into separate subsections.

3.1. Corresponding surfaces to CD production functions. Let $Q$ be a generalized CD production function of 2 variables given by

$$
Q: \mathbb{R}_{+}^{2} \longrightarrow \mathbb{R}_{+}, \quad(k, l) \longmapsto Q(k, l)=A k^{\alpha} l^{\beta}, \quad A, \alpha, \beta>0,
$$

where $k$ and $l$ respectively denotes the capital input and the labor input. 
From (3.1), the generalized CD production function has constant return to scale if and only if $\alpha+\beta=1$. Further, it has increasing return to scale (resp. decreasing return to scale) if and only if $\alpha+\beta>1$ (resp. $\alpha+\beta<1$ ).

For the generalized CD production given by (3.1), the elasticities of production with respect to $k$ and $l$ are respectively

$$
E_{k}=\frac{\partial Q / \partial k}{Q / k}=\alpha \quad \text { and } \quad E_{l}=\frac{\partial Q / \partial l}{Q / l}=\beta .
$$

The corresponding graph surface in $\mathbb{G}_{3}$ to the generalized CD production function is parameterized by

which called Cobb-Douglas (CD) surface.

$$
\mathbf{r}(k, l)=\left(k, l, A k^{\alpha} l^{\beta}\right),
$$

The Gaussian curvature of the CD surface is of the form

$$
K=\frac{Q^{2} \alpha \beta}{k^{2} l^{2}} \frac{1-(\alpha+\beta)}{\left(1+\left(\frac{\beta}{l} Q\right)^{2}\right)^{2}} .
$$

From (3.3), if the CD surface has vanishing Gaussian curvature $(K=0)$, then $Q$ has constant return to scale. Moreover, the last equality implies that $Q$ has increasing return to scale (resp. decreasing return to scale) if and only if the CD surface has negative (resp. positive) the Gaussian curvature.

Theorem 3.1. For a generalized CD production function $Q$, we have the following:

(A) $Q$ has constant return to scale if and only if the CD surface has vanishing Gaussian curvature in $\mathbb{G}_{3}$,

(B) $Q$ has increasing return to scale (resp. decreasing return to scale) if and only if the CD surface has negative (resp. positive) Gaussian curvature in $\mathbb{G}_{3}$.

Remark 3.1. Theorem 3.1 is the Galilean version of the Theorem 3.1 of [33]. Also statement (A) of Theorem 3.1 is a special case of the Theorem A of [14].

Now we investigate the minimality of the CD surfaces in $\mathbb{G}_{3}$. For this purpose, the mean curvature of the CD surface is given by

$$
H=\frac{A \beta(\beta-1) k^{\alpha} l^{\beta-2}}{2\left(1+\left(\frac{\beta}{l} Q\right)^{2}\right)^{\frac{3}{2}}} .
$$

If the CD surface is minimal, then we have from (3.4), $\beta=1$. This yields from (3.2) that the generalized CD production function has constant elasticity of production 1 with respect to the input $l$.

Also, it follows from (3.4) that the CD surface has positive (resp. negative) mean curvature if and only if the generalized CD production function has constant elasticity of production $E_{l}<1$ (resp. $E_{l}>1$ ) with respect to the input $l$.

Theorem 3.2. For a generalized $C D$ production function $Q$, we have the following statements: 
(A) $Q$ has constant elasticity of production $E_{l}=1$ with respect to the labor input if and only if the corresponding $C D$ surface in $\mathbb{G}_{3}$ is minimal;

(B) $Q$ has constant elasticity of production $E_{l}=\beta, 0<\beta<1$ (resp. $\beta>1$ ) with respect to the labor input if and only if the corresponding $C D$ surface $\mathbb{G}_{3}$ has positive (resp. negative) mean curvature.

A minimal CD surface corresponds to the generalized CD production function of the form $Q(k, l)=A k^{\alpha} l$. So it always has the increasing return to scale since $\alpha>0$.

Corollary 3.1. A generalized CD production function has always increasing return to scale if the corresponding $C D$ surface in $\mathbb{G}_{3}$ is minimal.

Remark 3.2. X. Wang and Y. Fu [36] obtained a non-existence result for the minimal $\mathrm{CD}$ hypersurfaces in $\mathbb{E}^{n+1}$. In our framework, this does not hold, i.e. there exist minimal CD surfaces in $\mathbb{G}_{3}$.

Remark 3.3. Since $\alpha, \beta>0$ for a generalized CD production function, there does not exist a CD surface of both curvature 0 in $\mathbb{G}_{3}$.

3.2. Corresponding surfaces to ACMS production functions. The generalized form of the ACMS production function depending on 2-inputs is given by

$$
Q: \mathbb{R}_{+}^{2} \longrightarrow \mathbb{R}_{+}, \quad(k, l) \longmapsto Q(k, l)=A\left(\alpha^{\rho} k^{\rho}+\beta^{\rho} l^{\rho}\right)^{\frac{\gamma}{\rho}},
$$

where $A>0, \rho<1, \rho \neq 0, \alpha, \beta, \gamma>0$ and $k, l$ are the capital input and the labor input, respectively.

Since the generalized ACMS production function $Q$ is homogeneous of degree $\gamma$, the elasticity of scale of $Q$ is $\gamma$.

The corresponding surface to the generalized ACMS production function is of the form

which we call $A C M S$ surface.

$$
\mathbf{r}(k, l)=\left(k, l, A\left(\alpha^{\rho} k^{\rho}+\beta^{\rho} l^{\rho}\right)^{\frac{\gamma}{\rho}}\right)
$$

The Gaussian curvature of such a surface is of the form

$$
K=\frac{A^{2} \gamma^{2} a^{\rho} \beta^{\rho}(k l)^{\rho-2}(\rho-1)\left(\alpha^{\rho} k^{\rho}+\beta^{\rho} l^{\rho}\right)^{2 \frac{\gamma}{\rho}-2}(\gamma-1)}{\left(1+\left(A \gamma \beta^{\rho} l^{\rho-1}\left(\alpha^{\rho} k^{\rho}+\beta^{\rho} l^{\rho}\right)^{\frac{\gamma}{\rho}-1}\right)^{2}\right)^{2}} .
$$

From (3.5), we get the following result.

Theorem 3.3. For a generalized ACMS production function $Q$, we have the following:

(A) $Q$ has constant return to scale if and only if the ACMS surface has vanishing Gaussian curvature $(K=0)$ in $\mathbb{G}_{3}$,

(B) $Q$ has increasing return to scale (resp. decreasing return to scale) if and only if the ACMS surface has negative (positive) the Gaussian curvature in $\mathbb{G}_{3}$.

Remark 3.4. Theorem 3.3 is the Galilean version of the Theorem 3.2 of [34]. Also statement (A) of Theorem 3.3 is a special case of Theorem A of [14]. 
The mean curvature of the ACMS surface is

$$
H=\frac{A \gamma \beta^{\rho} l^{\rho-2}\left(\alpha^{\rho} k^{\rho}+\beta^{\rho} l^{\rho}\right)^{\frac{\gamma}{\rho}-2}\left((\rho-1) \alpha^{\rho} k^{\rho}+(\gamma-1) \beta^{\rho} l^{\rho}\right)}{2\left(1+\left(A \gamma \beta^{\rho} l^{\rho-1}\left(\alpha^{\rho} k^{\rho}+\beta^{\rho} l^{\rho}\right)^{\frac{\gamma}{\rho}-1}\right)^{2}\right)^{\frac{3}{2}}} .
$$

Considering (3.6) gives that the ACMS surface is minimal if and only if $\rho=\gamma=1$. This yields that the generalized ACMS production function is a perfect substitute, i.e. is the form of

$$
Q(k, l)=\alpha_{1} k+\alpha_{2} l
$$

for some positive constants $\alpha_{1}, \alpha_{2}$. Therefore we have the following.

Theorem 3.4. A generalized ACMS production function is a perfect substitute if and only if the $A C M S$ surface in $\mathbb{G}_{3}$ is minimal.

From (3.6), if $\gamma<1$ then the ACMS surface has negative mean curvature since $\rho<1$. Hence the next result can be given.

Corollary 3.2. A generalized ACMS production function has decreasing return to scale if and only if the ACMS surface has negative mean curvature.

3.3. Corresponding surfaces to Allen production functions. An Allen production function with 2-inputs is defined by [20]

$$
Q: \mathbb{R}_{+}^{2} \longrightarrow \mathbb{R}_{+}, \quad(k, l) \longmapsto Q(k, l)=A\left(\alpha k^{2}+2 \beta k l+\gamma l^{2}\right)^{\frac{1}{2}},
$$

where $A, \alpha, \beta, \gamma>0$, and $k$ and $l$ respectively denotes the capital input and the labor input.

The corresponding surface to the Allen production function is of the form

$$
\mathbf{r}(k, l)=\left(k, l, A\left(\alpha k^{2}+2 \beta k l+\gamma l^{2}\right)^{\frac{1}{2}}\right),
$$

which we call Allen surface.

Remark 3.5. Note that the Allen production function $Q$ is homogeneous of degree 1 and has constant return to scale. Moreover it is easily seen that $Q$ satisfies the homogeneous Monge-Ampère equation $Q_{k k} Q_{l l}-Q_{k l}^{2}=0$ and thus the Allen surface in $\mathbb{G}_{3}$ is flat.

Now, let us assume that the Allen surface is minimal in $\mathbb{G}_{3}$. Thus, we derive from $(2.2)$

$$
0=\frac{\left(\alpha \gamma-\beta^{2}\right) k^{2}}{\alpha k^{2}+2 \beta k l+\gamma l^{2}}
$$

and $\alpha \gamma-\beta^{2}=0$. Then it follows from (3.7) that the Allen production function $Q$ can be rewritten as

$$
Q(k, l)=A(\lambda x+\mu y), \quad \lambda, \mu>0,
$$

which yields that $Q$ is a perfect substitute. 
Theorem 3.5. An Allen production function is a perfect substitute if and only if the corresponding surface is minimal in $\mathbb{G}_{3}$.

\section{REFERENCES}

[1] R. G. Allen, J. R. Hicks, A reconsideration of the theory of value Pt. II, Economica 1 (1934), 196-219.

[2] K. J. Arrow, H. B. Chenery, B. S. Minhas, R. M. Solow, Capital-labor substitution and economic efficiency, Rev. Econom. Stat. 43(3) (1961), 225-250.

[3] M. E. Aydin, M. Ergut, Homothetic functions with Allen's perspective and its geometric applications, Kragujevac J. Math. 38(1) (2014), 185-194.

[4] M. E. Aydin, M. Ergut, Composite functions with Allen determinants and their applications to production models in economics, Tamkang J. Math. 45(4) (2014), 427-435.

[5] M. E. Aydin, M. Ergut, Hessian determinants of composite functions with applications for production functions in economics, Kragujevac J. Math. 38(2) (2014), 259-268.

[6] M. E. Aydin, A. Mihai, Classifications of quasi-sum production functions with Allen determinants, Filomat 29(6) (2015), 1351-1359.

[7] M. E. Aydin, A. O. Ogrenmis, M. Ergut, Classification of factorable surfaces in the pseudoGalilean space, Glas. Mat. Ser. III, to appear.

[8] R. G. Chambers, Applied Production Analysis, Cambridge University Press, 1998.

[9] B.-Y. Chen, G. E. Vîlcu, Geometric classifications of homogeneous production functions, Appl. Math. Comput. 225 (2013), 345-351.

[10] B.-Y. Chen, S. Decu, L. Verstraelen, Notes on isotropic geometry of production models, Kragujevac J. Math. 38(1) (2014), 23-33.

[11] B.-Y. Chen, On some geometric properties of h-homogeneous production function in microeconomics, Kragujevac J. Math. 35(3) (2011), 343-357.

[12] B.-Y. Chen, On some geometric properties of quasi-sum production models, J. Math. Anal. Appl. 392 (2012), 192-199.

[13] B.-Y. Chen, Classification of h-homogeneous production functions with constant elasticity of substitution, Tamkang J. Math. 43(2) (2012), 321-328.

[14] B.-Y. Chen, A note on homogeneous production models, Kragujevac J. Math. 3(1) (2012), 41-43.

[15] B.-Y. Chen, Solutions to homogeneous Monge-Ampere equations of homothetic functions and their applications to production models in ecenomics, J. Math. Anal. Appl. 411(1) (2014), 223-229.

[16] C. W. Cobb, P. H. Douglas, A theory of production, Amer. Econom. Rev. 18(1) (1928), 139-165.

[17] S. Decu, L. Verstraelen, A note on the isotropical geometry of production surfaces, Kragujevac J. Math. 37(2) (2013), 217-220.

[18] M. Dede, Tubular surfaces in Galilean space, Math. Commun. 18(1) (2013), 209-217.

[19] Z. Erjavec, On generalization of helices in the Galilean and the pseudo-Galilean space, J. Math. Res. 6(3) (2014), 39-50.

[20] A. C. Ioan, A study of Allen production function with differential geometry, Acta Universitatis, Danubius 11(1) (2015), 115-119.

[21] M. K. Karacan, Y. Tuncer, Tubular surfaces of Weingarten types in Galilean and pseudo-Galilean, Bull. Math. Anal. Appl. 5(2) (2013), 87-100.

[22] P. O. Lindberg, E. A. Eriksson, L. G. Mattsson, Homothetic functions revisited, Econ. Theory 19(2) (2002), 417-427.

[23] L. Losonczi, Production functions having the CES property, Acta Math. Acad. Paedagog. Nyhái. (N.S.) 26(1) (2010), 113-125.

[24] S. K. Mishra, A brief history of production functions, IUP J. Manage. Econom. 8(4) (2010), $6-34$. 
[25] A. O. Ogrenmis, M. Ergut, M. Bektas, On the helices in the Galilean Space G, Iranian J. Sci. Tech., 31(A2) (2007), 177-181.

[26] A. Onishchick, R. Sulanke, Projective and Cayley-Klein Geometries, Springer, 2006.

[27] H. B. Oztekin, S. Tatlipinar, On some curves in Galilean plane and 3-dimensional Galilean space, J. Dyn. Syst. Geom. Theor. 10(2) (2012), 189-196.

[28] Z. M. Sipus, Ruled Weingarten surfaces in the Galilean space, Period. Math. Hungar. 56 (2008), 213-225.

[29] Z. M. Sipus, B. Divjak, Translation surface in the Galilean space, Glas. Mat. Ser. III 46(2) (2011), 455-469.

[30] Z. M. Sipus, B. Divjak, Surfaces of constant curvature in the pseudo-Galilean space, Int. J. Math. Sci. (2012), Article ID 375264, 28 pages.

[31] A. Thompson, Economics of the Firm, Theory and Practice, 3rd edition, PrenticeHall, 1981.

[32] H. Uzawa, Production functions with constant elasticities of substitution, The Review of Economic Studies 29(4) (1962), 291-299.

[33] G. E. Vîlcu, A geometric perspective on the generalized Cobb-Douglas production functions, Appl. Math. Lett. 24 (2011), 777-783.

[34] A. D. Vilcu, G. E. Vilcu, On some geometric properties of the generalized CES production functions, Appl. Math. Comput. 218 (2011), 124-129.

[35] A. D. Vilcu, G. E. Vilcu, On homogeneous production functions with proportional marginal rate of substitution, Math. Probl. Eng. (2013), Article ID 732643, 5 pages.

[36] X. Wang, Y. Fu, Some characterizations of the Cobb-Douglas and CES production functions in microeconomics, Abstract Appl. Anal. doi.org/10.1155/2013/761832.

[37] D. W. Yoon, Some classification of translation surfaces in Galilean 3-space, Int. J. Math. Anal. 6(28) (2012), 1355-1361.

${ }^{1}$ Department of Mathematics,

Firat UNIVERSITY,

23119, Elazig, Turkey

E-mail address: meaydin@firat.edu.tr

${ }^{2}$ Department of Mathematics,

Ahi Evran University, 40100, Kirsehir, TURKEY

E-mail address: sezinaykurt@hotmail.com 